psychopraxis. neuropraxis $2018 \cdot 21: 265-271$ https://doi.org/10.1007/s00739-018-0519-6 Online publiziert: 14. September 2018 (c) Der/die Autor(en) 2018

CrossMark

\section{Peter Berger}

Klinische Abteilung für Sozialpsychiatrie, Medizinische Universität Wien, Wien, Österreich

\section{Glücksspielabhängigkeit}

\section{Kasuistik und Diskussion einer Verhaltensabhängigkeit}

Etwa ein Prozent der Erwachsenen leidet in ihrem Leben an problematischen Glücksspielverhalten. Es entwickelt sich dabei ein immer stärkerer Drang zu spielen, dem nicht mehr widerstanden werden kann. Das Spielen beherrscht das Leben und führt in der Regel zu massiven finanziellen, familiären, psychischen oder auch rechtlichen Problemen. Es geht nicht allein darum Geld zu gewinnen, sondern auch darum, durch die Wirkungen des Spiels die eigene Befindlichkeit $\mathrm{zu}$ verbessern.

Nur weniger als $10 \%$ der von der Glücksspielabhängigkeit Betroffenen suchen primär wegen dieser Erkrankung Hilfe auf. Hinter mancher schwer zu behandelnden Depression oder Angststörung kann sich eine Glücksspielabhängigkeit verbergen, die durch die enormen finanziellen und psychischen Belastungen einen wichtigen Stressfaktor darstellt. Da diese Krankheit in der Regel verheimlicht wird, ist sie oft eine schwierige diagnostische Herausforderung. Die folgende Kasuistik soll dies verdeutlichen.

\section{Fallbericht}

Ein 37-jähriger Patient sucht ambulante Therapie wegen Problemen mit Glücksspielsucht auf. Er habe mit 20 Jahren begonnen, an Glücksspielautomaten zu spielen. Freunde hätten ihn damals mitgenommen und das Spiel habe ihm Spaß gemacht, er habe gesehen, wie andere Geld gewonnen hätten. In den weiteren Monaten sei er auch immer häufiger allein spielen gegangen. Er habe in dieser Zeit auch selbst einen für seine finanziellen Verhältnisse großen Gewinn gemacht, der sich in sein Gedächtnis eingeprägt habe. Er habe dann regelmäßig gespielt, und es habe immer häufiger Monate gegeben, in denen er seinen gesamten Lohn verspielt habe. Das Verlangen zu spielen sei vor allem nach einem anstrengenden Arbeitstag aufgetreten, und er habe während des Spiels sofort „abschalten" können. Er habe in dieser Zeit zwar auch anderen Interessen und immer der Arbeit nachgehen können, aber häufig sei ein starker Drang zu spielen aufgetreten, der ihm „wie magnetisch“ zum Glücksspiel gezogen hätte. Alkohol habe er während des Glücksspiels nie getrunken. Auch sonst trinke er nur selten und mäßig Alkohol.

Vor etwa 3 Jahren, im Rahmen von beruflichen und persönlichen Belastungen, habe das Glücksspiel bei ihm an Intensität zugenommen. Er sei mindestens 3-mal pro Woche spielen gegangen und habe das Casino häufig erst in der Früh verlassen, typischerweise erst, als er kein Geld mehr zur Verfügung hatte. Er habe auch während der Arbeit oft ans Glücksspiel gedacht, besonders daran, wie er Verluste durch neuerliches Glücksspiel ausgleichen könnte. Um das Spielen zu finanzieren, habe er Ersparnisse aufgelöst, das Konto überzogen und einen Kredit aufgenommen. Er habe sich „wie ferngesteuert“ gefühlt und es habe ihn zum Glücksspiel hingezogen, obwohl ihm bewusst gewesen sei, dass es ihm nicht gut tat. Er habe nun an Schlafstörungen und Reizbarkeit gelitten. Er sei sonst im Alltag und bei der Arbeit ein kontrollierter und besonnener Mensch. Daher sei ihm nun erstmals bewusst geworden, dass das Glücksspiel bei ihm außer Kontrolle geraten war. Er habe sich Bücher zum Thema Glücksspielabhängigkeit besorgt und gelesen und habe sich darin in seinem Problem bestätigt gefunden. In einem Casino habe er sich sperren lassen, er sei jedoch auf andere Möglichkeiten ausgewichen und ins nahe Ausland gefahren, um weiter spielen $\mathrm{zu}$ können. Vor etwa 2 Jahren habe er erstmals einen Arzt wegen Schlafstörungen aufgesucht. Dieser habe ihn auch gefragt, ob er besondere Belastungen habe.

\section{》) Glücksspielanhängigkeit ist sehr schambesetzt und wird daher meist verheimlicht}

Er habe sich jedoch nicht getraut, über seine Glücksspielabhängigkeit zu sprechen. Das verordnete Zolpidem habe er nur an wenigen Tagen genommen, da er befürchtet habe, sonst davon abhängig zu werden.

Im letzten Jahr, bevor er sich in Behandlung begeben hatte, sei sein psychischer Zustand zunehmend schlechter geworden: Er habe an Ein- und Durchschlafstörungen gelitten, unkontrolliert gegessen und $15 \mathrm{~kg}$ zugenommen, sich bei der Arbeit immer schlechter konzentrieren können und sich energielos und freudlos gefühlt, er sei leicht reizbar gewesen und habe täglich ans Glücksspiel gedacht, wobei ihn dies belastete, zeitweise seien auch Suizidideen ohne Konkretisierung aufgetreten. Bevor er sich in Behandlung begeben hatte, habe er sich gesagt: „Wenn ich jetzt noch einmal Spielen gehe, obwohl ich es eigentlich nicht will, dann suche ich Hilfe auf."

Bei Behandlungsbeginn war der Patient deutlich depressiv verstimmt mit der oben erwähnten Symptomatik. Zusätzlich litt er auch an mehrfach während des Tages auftretenden, etwa eine Minute dauernden Vorstellungskonkre- 
Wiederholte Episoden von Glücksspiel

Diese Episoden bringen den Betroffenen keinen Gewinn, sondern werden trotz subjektivem Leidensdruck und Störung der sozialen und beruflichen Funktionsfähigkeit fortgesetzt

Intensiver Drang, zu spielen, der nur schwer kontrolliert werden kann. Ist nicht in der Lage, das Glücksspiel durch Willensanstrengung zu unterbrechen

Ständige Beschäftigung mit Gedanken oder Vorstellungen vom Glücksspiel oder mit dem Umfeld des Glücksspiels

DSM-5 (Störung durch Glücksspiel) [3]

A. Andauerndes und wiederkehrendes fehlangepasstes Spielverhalten, was sich in mindestens vier der folgenden Merkmale ausdrückt:

1. Muss mit immer höheren Einsätzen spielen, um die gewünschte Erregung zu erreichen

2. Ist unruhig und gereizt beim Versuch, das Spielen einzuschränken oder aufzugeben

3. Wiederholte erfolglose Versuche, das Spielen zu kontrollieren, einzuschränken oder aufzugeben

4. Ist stark eingenommen vom Glücksspiel

5. Häufiges Glücksspielen in belastenden Gefühlszuständen

6. Kehrt nach Verlust oft am nächsten Tag zurück, um diesen auszugleichen (dem Verlust hinterher jagen)

7. Belügt andere, um das Ausmaß seiner Verstrickung in das Spielen zu vertuschen

8. Hat eine wichtige Beziehung, seinen Arbeitsplatz, Ausbildungs- oder Aufstiegschancen wegen des Spielens gefährdet oder verloren

9. Verlässt sich darauf, dass andere ihm Geld bereitstellen, um die durch das Spielen verursachte hoffnungslose finanzielle Situation zu überwinden

B. Kann nicht durch manische Episode erklärt werden

DSM Diagnostic and Statistical Manual of Mental Disorders, ICD-10 International Classification of Diseases

tisierungen, in denen er Glücksspielerinnerungen wie Flashbacks sehr plastisch wiedererlebte. Diese Episoden erlebte der Patient als sehr unangenehm. Bereits eine Woche vor Behandlungsbeginn, seit dem Entschluss, konkret Hilfe zu suchen, war der Patient glücksspielabstinent.

Die medikamentöse Behandlung erfolgte mit Trazodon abends, da die depressive Symptomatik mit ausgeprägten Schlafstörungen im Vordergrund stand. Acht Wochen nach Behandlungsbeginn war der Patient bezüglich der depressiven Symptomatik und der Schlafstörungen deutlich gebessert und weiter abstinent. Auch die Flashbacks traten deutlich seltener auf und waren weniger angstbesetzt.

\section{Diskussion}

Das Bedürfnis nach Glücksspielen ist in Ländern, in denen das Glücksspiel verfügbar ist, weit verbreitet. In Österreich nimmt einer rezenten Untersuchung [1, 2] zufolge etwa die Hälfte der Erwachsenen Bevölkerung an Glücksspielen teil. Bei $1,1 \%$ führt das Glücksspiel zu ausgeprägten Problemen, etwa 0,6-0,7 \% erfüllen ausreichend Kriterien für die Diagnose einer Glücksspielabhängigkeit oder „Störung durch Glücksspiel/ pathologisches Glücksspiel“" wie diese
Erkrankung nach den offiziellen diagnostischen Manualen DSM-5 (Diagnostic and Statistical Manual of Mental Disorders; [3]) bzw. ICD-10 (International Classification of Diseases; [4]) bezeichnet wird.

Die Glücksspielabhängigkeit ist nicht allein durch häufiges Spielen gekennzeichnet, sondern durch die zunehmende gedankliche Einengung auf das Glücksspiel. Das Glücksspiel wird zum Wichtigsten im Leben, dadurch wird dafür nicht nur viel Geld ausgegeben, sondern auch viel Zeit damit verbracht, Zeit, die dem Familienleben, anderen Interessen, der Arbeit oder auch sozialen Beziehungen verloren geht.

Die Betroffenen erleben einen plötzlichen Drang zu spielen, der scheinbar ohne Anlass auftritt und als überwältigend und unwiderstehlich erlebt wird. Sie können dann an nichts anderes mehr denken und erleben innere Unruhe (meist auch mit vegetativen Symptomen), die sich erst durch das Glücksspiel löst.

Spieler fühlen sie sich wie in einer anderen Welt, sie fühlen sich wohl und befreit von Sorgen. Typischerweise kann das Spiel erst beendet werden, wenn kein Geld mehr verfügbar ist. Das Spiel endet bei Glücksspielsüchtigen somit fast immer mit Verlust, auch wenn zwischen- zeitlich Gewinne aufgetreten sind. Die Verluste führen dazu, dass das Bedürfnis entsteht, verlorenes Geld demnächst wieder zurückzugewinnen, wodurch sich das Verhalten perpetuiert. Als finanzielle Folge der Spielsucht entstehen fast immer Schulden. Der finanzielle Druck trägt dazu bei, dass weiter und mit höheren Einsätzen gespielt wird, da der Gedanke auftritt, dass nur ein großer Gewinn die finanziellen Probleme lösen kann.

Sehr typisch ist auch, dass das Ausmaß des Spielverhaltens, aber auch das der Folgen verheimlicht wird. Einerseits wird verheimlicht und gelogen, um trotz der massiven Folgen Geld zu bekommen und weiterspielen zu können oder um Schuldner zu vertrösten, aber auch, weil das Spielen sehr schambesetzt sein kann oder auch die (berechtigte) Angst besteht, dass bei anderen kein Verständnis dafür besteht, dass man so ,verrückt“ sein kann, trotz massiver Probleme weiterzuspielen. Gerade das Lügen bezüglich des Glücksspielverhaltens erleben viele Betroffene als massive Belastung und als besorgniserregende Charakterveränderung.

\section{Wirkung des Glücksspiels}

Obwohl es beim Glücksspiel um Gewinn oder Verlust von Geld geht, ist bei der 
Hier steht eine Anzeige.

\section{曾 Springer}


Glücksspielabhängigkeit der Wunsch Geld zu gewinnen nur ein Aspekt des Glücksspiels. Viel stärker ist seine emotionale Wirkung. Bereits der Gedanke an das Spiel bewirkt eine Verbesserung der Befindlichkeit, Sorgen treten in den Hintergrund und die Erwartungshaltung (bezüglich Gewinne) wird optimistisch.

Während des Spiels bewirkt die Anspannung durch die Erwartung des Spielergebnisses eine ständige Stimulierung des Belohnungssystems. Auch der in der Kasuistik beschriebene Patient berichtete, dass er sich wie in einer anderen Welt und frei von alläglichen Sorgen fühlte und dass Spielen die beste Art sei, sich zu entspannen.

Durch die finanziellen Verluste aufgrund des Kontrollverlustes folgt depressive Verstimmung, die in Zusammenwirken mit dem Wunsch, die Verluste wieder auszugleichen, zu weiterem Drang $\mathrm{zu}$ spielen führt. So entsteht eine Spirale von positiven und negativen Verstärkern, und das Spielen verankert sich bei der Glücksspielabhängigkeit als letztendlich dysfunktionale, aber für die Patienten unmittelbar beste und einfachste Art der emotionalen Regulation.

\section{Diagnostik und Komorbidität}

In beiden diagnostischen Systemen (ICD-10 und DSM-5; [3, 4]) beschreiben die diagnostischen Kriterien neben der exzessiven Häufigkeit des Spielens die ständige gedankliche Beschäftigung mit dem Glücksspiel, den intensiven Drang zu spielen, dem nicht widerstanden werden kann, die Unfähigkeit, das Spielen selbst zu beenden, und das fortgesetzte Spielen trotz gravierender Folgen (• Tab. 1).

Im DSM-5 werden aber noch weitere typische Verhaltensweisen angeführt, wie das Lügen bezüglich der Häufigkeit des Glücksspiels und daraus resultierenden Verlusten, und dem Drang, Verluste durch neuerliches Spielen wieder auszugleichen. Zusätzlich gibt es Kriterien, die denen der substanzgebundenen Abhängigkeiten entsprechen, wie wiederholte erfolglose Versuche das Spielen einzuschränken oder aufzugeben, das Spielen, um Problemen zu entkommen oder um die Stimmung zu verbessern (Wirkung),

psychopraxis. neuropraxis $2018 \cdot 21: 265-271 \quad$ https://doi.org/10.1007/s00739-018-0519-6

c) Der/die Autor(en) 2018

\section{P. Berger \\ Glücksspielabhängigkeit. Kasuistik und Diskussion einer Verhaltensabhängigkeit}

\section{Zusammenfassung}

Der präsentierte Fall zeigt, dass sich nach jahrelangem problematischem Glücksspielverhalten, bei dem das Glücksspiel vom Patienten positiv und als wirksames Mittel zur Spannungsregulation erlebt wird, eine Glücksspielabhängigkeit entwickeln kann. Typisch für diese erste Phase ist, dass kein ausreichendes Problembewusstsein besteht, obwohl bereits einzelne Probleme bestehen. Auch in der Phase der ausgeprägten Abhängigkeit entwickelt sich nur langsam Krankheitseinsicht, und die Motivation zur Behandlung ist ambivalent. Die zunehmende psychische Belastung führt dann dazu, dass das Glücksspiel zunehmend als negativ und belastend erlebt wird. Daraus ergeben sich oft erst die entsprechende Krankheitseinsicht und Motivation zur Behandlung. Trotzdem bleibt die Problematik schambesetzt. Daher sollte als erster Behandlungsschritt den Patienten das nötige Verständnis für ihre Erkrankung vermittelt werden.

\section{Schlüsselwörter}

Glücksspiel-Abhängigkeit · Problematisches Glücksspielverhalten · Motivation Krankheitseinsicht · Depression

\section{Gambling Addiction. Case Study and Discussion of Behavioral Addiction}

\section{Abstract}

The presented case shows that gambling addiction can develop after many years of problematic gambling behavior in which gambling is experienced by the patient as a positive and effective means of tension regulation. Typical for this initial phase is that a sufficient perception of the problem does not exist although some problems already exist. Even during the phase of advanced dependence, insight into the disease only slowly develops and the motivation for treatment is ambivalent. The increasing mental stress then leads to the fact that gambling is increasingly experienced as negative and burdensome. This often first results in the appropriate insight into the disease and motivation for treatment. Nevertheless, the problem remains surrounded by shame. Therefore, the first treatment step should be to provide the patient with the necessary understanding of the disease.

\section{Keywords}

Pathological gambling · Problem gambling behavior · Motivation · Insight · Depression das Steigern von Einsätzen, um die gewünschte Erregung zu erreichen (Toleranzentwicklung), und Unruhe und Gereiztheit beim Versuch, das Spielen einzuschränken (Entzugssymptome).

Gestützt auf Untersuchungen, die u. a. auf gemeinsame genetische Faktoren und ähnliche Aktivierungsmuster bei Substanzabhängigkeit und Spielsucht hinweisen, ist im DSM-5 die Glücksspielabhängigkeit als „Abhängigkeitsstörung ohne Substanzbezug" verzeichnet, was auch in der zukünftigen Version der ICD (ICD-11) geplant ist.

Bei dem in der Kasuistik vorgestellten Patienten zeigt sich, dass sich die Störung über Jahre progredient entwickelte. In den ersten Jahren treffen nur einzel- ne Symptome zu; insbesondere besteht ein unwiderstehlicher Drang zu spielen, aber Folgeprobleme lassen sich bewältigen. In den letzten 3 Jahren eskaliert das Verhalten und es treffen alle (ICD-10) bzw. die meisten (DSM-5) der diagnostischen Symptome zu.

Die meisten Glücksspielabhängigen leiden zusätzlich an anderen psychiatrischen Erkrankungen. Das Erkennen dieser Erkrankungen ist für die Planung der weiteren Behandlung wichtig. In epidemiologischen Studien zeigt sich eine sehr hohe Komorbidität mit substanzbezogenen Abhängigkeiten, affektiven Erkrankungen sowie Angst- und Persönlichkeitsstörungen [5]. Bei Patienten, die primär wegen Spielsucht Hilfe auf- 
suchen, findet sich meist eine deutlich geringere Rate von substanzbezogenen Abhängigkeiten. Eine Depression kann sowohl primär bestehen als auch sekundär im Rahmen der zunehmenden Belastungen durch die Spielsucht auftreten. In beiden Fällen verstärkt dabei die negative Befindlichkeit den Drang zu spielen und den Wunsch, damit die Stimmung zu verbessern. Bei dem hier vorgestellten Patienten finden sich in der Anamnese keine psychiatrischen Vorerkrankungen, sondern es entwickelt sich bei ihm im Rahmen der Belastungen eine ausgeprägte Depression, die ihn letztendlich zur Behandlung führte.

\section{Behandlung}

Der erste Schritt in der Behandlung ist die Klärung und Förderung der Krankheitseinsicht und der Änderungsmotivation, die meist ambivalent ist. Der hier vorgestellte Patient hatte sich bereits vor Behandlungsbeginn mit beiden auseinandergesetzt, was prognostisch günstig ist, aber sehr häufig nicht der
Fall ist. Weitere Schritte können sein: die Empfehlung von Sicherungsmaßnahmen (z.B. nur wenig Geld bei sich zu haben), die Schuldenregulierung, die Analyse der persönlichen Funktionalität des Spielverhaltens und die Identifizierung von Auslöser für den Drang zu spielen.

Die medikamentöse Therapie orientiert sich in erster Linie an der Behandlung einer komorbiden Störung. Kontrollierte Studien zeigen, dass Opioidantagonisten wie Naltrexon das Suchtverlangen reduzieren können [6]. Diese sind aber in dieser Indikation nicht zugelassen, sondern können nur off-label eingesetzt werden. Beim in der Kasuistik vorgestellten Patienten wurde eine antidepressive Therapie mit Trazodon durchgeführt, da dies wegen der sedierenden Wirkung auch bezüglich der Schlafstörung günstig erschien. Da der Patient nach seiner Entscheidung zur Behandlung über keinen Drang zu spielen berichtete, sondern Erinnerungen an das Glücksspiel angstbesetzt waren, wurde Naltrexon nicht erwogen.

\section{Hilfesuchverhalten und Verheimlichung der Glücksspielabhängigkeit}

Auch wenn die negativen Folgen der Glücksspielabhängigkeit meist erst nach Jahren des Bestehens der Erkrankung zur Behandlung führen, haben sich gleichzeitig die verschiedenen positiv erlebten Wirkungen des Glücksspiels eingeprägt und die Problembewältigungsmechanismen der Betroffenen auf diese positiven Wirkungen des Glücksspiels eingeengt. Daher ist die Behandlungsmotivation in der Regel ambivalent, und Betroffene suchen im Durchschnitt erst nach etwa 8 Jahren Problemdauer erstmals Hilfe auf.

\section{I) Nur maximal 10\% der Glücksspielabhängigen suchen wegen ihrer Krankheit Hilfe auf}

Die wenigen Studien, die zum Hilfesuchverhalten von Glücksspielabhängigen vorliegen, zeigen, dass nur maximal

Hier steht eine Anzeige. 
Hier steht eine Anzeige. Springer
$10 \%$ der Betroffenen wegen dieser Abhängigkeit Hilfe aufsuchen [7].

Falls Patienten den Wunsch in sich hegen, mit dem Spielen aufzuhören, besteht oft große Scham davon zu erzählen, oder es wird erwartet, dass kein Verständnis für diese Problematik besteht. Daher ist es eine besondere Herausforderung, Spielsucht bei Patienten zu erkennen, die nicht primär wegen diesem Leiden zu Behandlung kommen, wie dies auch beim Patienten der Kasuistik der Fall war. Wie bereits erwähnt, leiden viele Glücksspielabhängige an Depressionen oder Angststörungen und suchen diesbezüglich eher Hilfe auf oder kommen wegen eines Suizidversuchs in Behandlung. Bei der Exploration psychosozialer Belastungsfaktoren können etwa ungeklärte finanzielle Probleme einen Hinweis auf eine bestehende Spielsucht ergeben.

\section{Fazit für die Praxis}

- Glücksspielabhängige suchen nicht nur wegen finanzieller Probleme oder auf Druck von Angehörigen Hilfe, sondern vor allem wegen psychischer Beschwerden. Das Glücksspiel ist dann nicht mehr Entspannung und Ablenkung von Problemen, sondern wird zur Belastung. Das Spielverhalten wird dadurch ichdyston, was die Krankheitseinsicht und die Motivation zur Änderung fördert.

- Glücksspielanhängigkeit ist sehr schambesetzt und wird daher meist verheimlicht, so auch im ärztlichen Gespräch, falls überhaupt ärztliche Hilfe aufgesucht wird. Hinter ungeklärten finanziellen Problemen kann sich eine Glücksspielabhängigkeit verbergen.

- Bei Komorbidität einer psychischen Erkrankung (wie z. B. Depression) und einer Glücksspielabhängigkeit sollte sich die Behandlung auf beide Erkrankungen richten.

\section{Korrespondenzadresse}

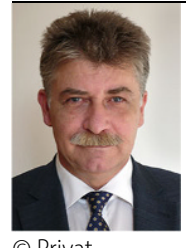

Ass. Prof. Dr. Peter Berger

Klinische Abteilung

für Sozialpsychiatrie,

Medizinische Universität

Wien

Währinger Gürtel 18-20,

1090 Wien, Österreich

peter.berger@meduniwien.ac.at

Funding. Open access funding provided by Medical University of Vienna.

\section{Einhaltung ethischer Richtlinien}

Interessenkonflikt. P. Berger gibt an, dass kein Interessenkonflikt besteht.

Dieser Beitrag beinhaltet keine von den Autoren durchgeführten Studien an Menschen oder Tieren.

Open Access. Dieser Artikel wird unter der Creative Commons Namensnennung 4.0 International Lizenz (http://creativecommons.org/licenses/by/4.0/deed de) veröffentlicht, welche die Nutzung, Vervielfältigung, Bearbeitung, Verbreitung und Wiedergabe in jeglichem Medium und Format erlaubt, sofern Sie den/die ursprünglichen Autor(en) und die Quelle ordnungsgemäßnennen, einen Link zur Creative Commons Lizenz beifügen und angeben, ob Änderungen vorgenommen wurden.

\section{Literatur}

1. Kalke J, Buth S, Rosenkranz M, Schütze C, Oechsler H, Verthein U (2011) Glücksspiel und Spielerschutz in Österreich. Empirische Erkenntnisse zum Spielverhalten der Bevölkerung und zurPrävention der Glücksspielsucht. Lambertus, Freiburgi. Brsg

2. Kalke J, Wurst FM (2015) Glücksspielverhalten und Glücksspielprobleme in Österreich. Ergebnisse einer Repräsentativbefragung. Institut für interdisziplinäre Sucht- und Drogenforschung, Hamburg

3. American Psychiatric Association (2015) Diagnostisches und Statistisches Manual Psychischer Störungen DSM-5. Hofgrefe, Göttingen (Deutsche Ausgabe herausgegeben von Peter Falkai und Hans-Ulrich Wittchen)

4. Dilling H, und Mitarbeiter (Hrsg) (1994) Internationale Klassifikation psychischer Störungen: ICD10, KapiteIV (F); Forschungskriterien/Weltgesundheitsorganisation, 1. Aufl. Huber, Bern, Göttingen, Toronto, Seattle

5. Petry NM, Stinson FS, Grant BF (2005) Comorbidity of DSM-IV pathological gambling and other psychiatric disorders: results from the National Epidemiologic Survey on Alcohol and Related Conditions. JClin Psychiatry 66(5):564-574

6. Mouaffak F, Leite C, Hamzaoui S, Benyamina A, Laqueille X, Kebir O (2017) Naltrexone in the treatment of broadly defined behavioral addictions: a review and meta-analysis of randomized controlled trials. Eur Addict Res 23(4):204-210

7. Slutske WS (2006) Natural recovery and treatmentseeking in pathological gambling: results of two U.S. national surveys. Am J Psychiatry 163(2):297-302 
Hier steht eine Anzeige.

\section{曾 Springer}

